\title{
Hermite-Hadamard Type Inequalities for $s$-Convex Stochastic Processes in the Second Sense
}

\author{
ERHAN SET, MUHARREM TOMAR*', SELAHATTIN MADEN \\ Department of Mathematics, Faculty of Science and Arts, Ordu University, Ordu, Turkey \\ *Corresponding author: muharremtomar@odu.edu.tr
}

Received September 24, 2014; Revised November 10, 2014; Accepted November 23, 2014

\begin{abstract}
In this study, s-convex stochastic processes in the second sense are presented and some well-known results concerning s-convex functions are extended to s-convex stochastic processes in the second sense. Also, we investigate relation between s-convex stochastic processes in the second sense and convex stochastic processes.
\end{abstract}

Keywords: Hermite Hadamard Inequality, s-convex functions, convex stochastic process, s-convex stochastic process

Cite This Article: ERHAN SET, MUHARREM TOMAR, and SELAHATTIN MADEN, "HermiteHadamard Type Inequalities for $s$-Convex Stochastic Processes in the Second Sense." Turkish Journal of Analysis and Number Theory, vol. 2, no. 6 (2014): 202-207. doi: 10.12691/tjant-2-6-3.

\section{Introduction}

First, the idea of convex functions was put forward, and many studies were made in this area. Later, in 1980, Nikodem [1] introduced the convex stochastic processes in his article. In 1995, Skowronski [3] presented some further results on convex stochastic processes. Moreover, in 2011, Kotrys [4] derived some Hermite-Hadamard type inequalities for convex stochastic processes. In 2014 Maden et. al. [7] introduced the convex stochastic processes in the first sense and extended some well-known results concerning convex functions in the first sense, such as Hermite-Hadamard type inequalities and some inequalities to convex stochastic processes.

We shall introduce the following definitions which are used throughout this paper.

Definition 1. $[1,2,8]$ Let $(\Omega, A, P)$ be an arbitrary probability space. A function $X: \Omega \rightarrow \mathbb{R}$ is called a random value if it is $A$ - measurable. A function $X: I \times \Omega \rightarrow \mathbb{R}$, where $I \subset \mathbb{R}$ is an interval, is called a stochastic process if for every $t \in I$ the function $X(t, \bullet)$ is a random variable.

Recall that the stochastic process $X: I \times \Omega \rightarrow \mathbb{R}$ is called

(1) continuous in probability in interval I, if for all $t_{0} \in I$

$$
P-\lim _{t \rightarrow t_{0}} X(t, \bullet)=X\left(t_{0}, \bullet\right)
$$

where $P$ - lim denotes the limit in probability;

(2) mean-square continuous in the interval I, if for all $t_{0} \in I$

$$
P-\lim _{t \rightarrow t_{0}} E\left[X(t, \bullet)-X\left(t_{0}, \bullet\right)\right]=0
$$

where $E[X(t, \bullet)]$ denotes the expectation value of the random variable $X(t, \bullet)$;

(3) increasing (decreasing) if for all $t, s \in I$ such that $<s$,

$$
X(t, \bullet) \leq X(s, \bullet),(X(t, \bullet) \geq X(s, \bullet)
$$

(4) monotonic if it is increasing or decreasing;

(5) differentiable at a point $t \in I$ if there is a random variable $X^{\prime}(t, \bullet): \Omega \rightarrow \mathbb{R}$

$$
X^{\prime}(t, \bullet)=P-\lim _{t \rightarrow t_{0}} \frac{X(t, \bullet)-X\left(t_{0}, \bullet\right)}{t-t_{0}}
$$

We say that a stochastic process $X: I \times \Omega \rightarrow \mathbb{R}$ is continuous (differentiable) if it is continuous (differentiable) at every point of the interval I.

Definition 2. $[2,3,8]$ Let $(\Omega, A, P)$ be a probability space and $I \subset \mathbb{R}$ be an interval. We say that a stochastic process $X: I \times \Omega \rightarrow \mathbb{R}$ is

(1) convex if

$$
X(\lambda s+(1-\lambda) t, \bullet) \leq \lambda X(s, \bullet)+(1-\lambda) X(t, \bullet)
$$

if all $s, t \in I$ and $\lambda \in[0,1]$. This class of stochastic process are denoted by $C$.

(2) $\lambda$-convex (where $\lambda$ is a fixed number from $(0,1))$ if

$$
X(\lambda s+(1-\lambda) t, \bullet) \leq \lambda X(s, \bullet)+(1-\lambda) X(t, \bullet)
$$

if all $s, t \in I$ and $\lambda \in(0,1)$. This class of stochastic process are denoted by $C_{\lambda}$.

(3) Wright-convex if

$X(\lambda s+(1-\lambda) t, \bullet)+X((1-\lambda) s+\lambda t, \bullet) \leq X(s, \bullet)+X(t, \bullet)$ 
if all $s, t \in I$ and $\lambda \in[0,1]$. This class of stochastic process are denoted by $W$.

(4) Jensen-convex if

$$
X\left(\frac{s+t}{2}, \bullet\right) \leq \frac{X(s, \bullet)+X(t, \bullet)}{2}
$$

This class of stochastic process are denoted by $\mathrm{C}_{1}$.

$$
\overline{2}
$$

Let $0<\mathrm{s} \leq 1$. A function $f: \mathbb{R}_{+} \rightarrow \mathbb{R}$ where $\mathbb{R}_{+}=[0, \infty)$, is said to be s-convex function in the second sense if:

$$
f(\alpha u+\beta v) \leq \alpha^{s} f(u)+\beta^{s} f(v)
$$

for all $\mathrm{u}, \mathrm{v} \geq 0$ with $\alpha+\beta=1$. This class of functions are denoted by $K_{s}^{2}$. It can be easily seen that for $\mathrm{s}=1 \mathrm{~s}-$ convexity in the first sense reduce to ordinary convexity of functions defined on $\mathbb{R}_{+}$.

Throughout this paper, let I be an interval on $\mathbb{R}_{+}$.

Now, we present some theorems which was proved by Dargomir and Fitzpatrick [5] and H. Hudzik and L. Maligranda [6] about some inequalities of HermiteHadamard type and basic properties for the s-convex functions in the second sense.

Proposition 1. [6] If $f \in K_{s}^{2}$, then $f$ is non-negatif on $[0, \infty)$.

Theorem 1. [6] Let $f \in K_{s}^{2}$. Then inequality (1.1) holds for all $u, v \in \mathbb{R}_{+}$and $\alpha, \beta \geq 0$ with $\alpha+\beta \leq 1$ if and only if $f(0)=0$.

Theorem 2. [5] Suppose that $f: \mathbb{R}^{+} \rightarrow \mathbb{R}^{+}$is s-convex mapping in the second sense, $s \in(0,1)$ and $a, b \in \mathbb{R}^{+}$with $a<b$. If $f \in L_{1}[a, b]$, then one has the inequalities:

$$
2^{s-1} f\left(\frac{a+b}{2}\right) \leq \frac{1}{b-a} \int_{a}^{b} f(x) d x \leq \frac{f(a)+f(b)}{s+1} .
$$

Theorem 3. [6] Now, suppose that $f$ is Lebesque integrable on $[a, b]$ and consider the mapping $H:[0,1] \rightarrow \mathbb{R}$ given by

$$
H(t):=\frac{1}{b-a} \int_{a}^{b} f\left(t x+(1-t) \frac{a+b}{2}\right) d x
$$

Let $f: I \subseteq \mathbb{R}_{+} \rightarrow \mathbb{R}$ be a s-convex mapping in the second sense on I, $s \in(0,1]$ and Lebesque integrable on $[a, b] \subseteq I, a<b$. Then:

$\mathrm{i}$ : $\mathrm{H}$ is s-convex in the second sense on $[0,1]$,

ii: We have the inequality:

$$
H(t) \geq 2^{s-1} f\left(\frac{a+b}{2}\right)
$$

for all $t \in[0,1]$.

iii: We have inequality:

$$
H(t) \leq \min \left\{H_{1}(t), H_{2}(t)\right\}, t \in[0,1]
$$

where

$$
\begin{aligned}
& H_{1}(t)=t^{s} \frac{1}{b-a} \int_{a}^{b} f(x) d x+(1-t)^{s} f\left(\frac{a+b}{2}, \bullet\right) \\
& H_{2}(t)=\frac{X\left(t x+(1-t) \frac{a+b}{2}, \bullet\right)+X\left(t b+(1-t) \frac{a+b}{2}, \bullet\right)}{s+1}
\end{aligned}
$$

and $t \in(0,1]$

iv: If $\widehat{H}(t, \bullet):=\max \left\{H_{1}(t), H_{2}(t)\right\}, t \in[0,1]$, then $\widehat{H}(t) \leq t^{s} \frac{f(a)+f(b)}{s+1}+(1-t)^{s} \frac{2}{s+1} f\left(\frac{a+b}{2}\right), t \in[0,1]$

Theorem 4. [6] Let $f: I \subseteq \mathbb{R}_{+} \rightarrow \mathbb{R}_{+}$be a s-convex mapping in the second sense, $s \in(0,1], a, b \in I$ with $a<b$ on $[a, b]$.

$$
F\left(s+\frac{1}{2}\right)=F\left(\frac{1}{2}-s\right)
$$

for all $s \in\left[0, \frac{1}{2}\right]$ and

$$
F(t)=F(1-t)
$$

for all $t \in[0,1]$;

ii. $\mathrm{F}$ is s-convex in the second sense on $[0,1]$;

iii. We have the inequality:

$$
\begin{aligned}
& 2^{1-s} F(t) \geq F\left(\frac{1}{2}\right)=\frac{1}{(b-a)^{2}} \int_{a}^{b} \int_{a}^{b} F\left(\frac{x+y}{2}\right) d x d y, \\
& t \in[0,1]
\end{aligned}
$$

iv. We have the inequality

$$
F(t) \geq 2^{s-1} H(t) \geq 4^{s-1} f\left(\frac{a+b}{2}\right)
$$

for all $t \in[0,1]$.

v. We have the inequality:

$$
F(t) \leq \min \left\{\begin{array}{l}
{\left[t^{s}+(1-t)^{s}\right] \frac{1}{b-a} \int_{a}^{b} f(x) d x,} \\
f(a)+f(t a+(1-t) b) \\
\frac{+f((1-t) a+t b)+f(b)}{(s+1)^{2}}
\end{array}\right\}
$$

for all $t \in[0,1]$.

The aim of this paper is to introduce convex stochastic processes in the second sense and to obtain basic properties and Hermite-Hadamard type inequalities for this procesess.

\section{2. s-convex Stochastic Process in the Second Sense}

Definition 3. $X: I \times \Omega \rightarrow \mathbb{R}$ stochastic process where $I \subset \mathbb{R}$ be an interval, is said to be s-convex in the second sense if: 


$$
X(\lambda u+(1-\lambda) v, \bullet) \leq \lambda^{s} X(u, \bullet)+(1-\lambda)^{s} X(v, \bullet)
$$

for all $u, v \geq 0$ and s fixed in $(0,1]$. We denote this class of stochastic process by $C_{s}^{2}$.

Remark 1. It can be easily seen that for $s=1 \mathrm{~s}$ convexity in the second sense for stochastic processes reduce to ordinary convexity of stochastic processes defined in Definition 2.

Proposition 2. $C \subseteq C_{s}^{2}$ for all $\lambda \in[0,1]$.

Proof. To prove the proposition assume that $X \in C$ and take arbitrary points $u, v \in I, s \in(0,1]$, Since $\mathrm{X}$ is convex stochastic process and $\lambda \leq \lambda^{S}$ on $[0,1]$ we have

$$
\begin{aligned}
X(\lambda u+(1-\lambda) v, \bullet) & \leq \lambda X(u, \bullet)+(1-\lambda) X(v, \bullet) \\
& \leq \lambda^{s} X(u, \bullet)+(1-\lambda)^{s} X(v, \bullet)
\end{aligned}
$$

Then $X \in C_{S}^{2}$.

Proposition 3. $C_{\frac{1}{2}} \subseteq C_{S}^{2}$ for all $\lambda \in(0,1)$.

Proof. To prove the proposition assume that $X \in C_{1}$ and take arbitrary points $u, v \in I, s \in(0,1]$, Since $\mathrm{X}$ is Jensen-convex stochastic process and $\frac{1}{2} \leq\left(\frac{1}{2}\right)^{S}$ we have

$$
X\left(\frac{u+v}{2}, \bullet\right) \leq \frac{X(u, \bullet)+X(v, \bullet)}{2} \leq \frac{X(u, \bullet)+X(v, \bullet)}{2^{s}}
$$

Then $X \in C_{S}^{2}$.

Proposition 4. $C \subset C_{\lambda} \subset C_{\frac{1}{2}} \subset C_{S}^{2}$ for all $\lambda \in(0,1)$.

Proof. The proof is cleared owing to [3, Proposition 3] and Proposition 3.

Proposition 5. If $X \in C_{S}^{2}$, then $X$ is non-negative on $I$.

Proof. We have, for $u \in I, s \in(0,1]$

$$
\begin{aligned}
& X(u, \bullet)=X\left(\frac{1}{2} u+\frac{1}{2} u, \bullet\right) \leq \frac{X(u, \bullet)}{2^{s}}+\frac{X(u, \bullet)}{2^{s}} \\
& =2^{1-s} X(u, \bullet)
\end{aligned}
$$

Hence, $\left(2^{1-s}-1\right) X(u, \bullet) \geq 0$ and so $X(u, \bullet) \geq 0$.

Theorem 5. Let $X \in C_{S}^{2}$. Then inequality $X(\alpha u+\beta v, \bullet) \leq \alpha^{s} X(u, \bullet)+\beta^{s} X(v, \bullet)$ holds for all $u, v \in I$ and $\alpha, \beta \geq 0$ with $\alpha+\beta \leq 1$ if and only if $\mathrm{X}(0, \bullet)=0$.

Proof. Necessity is obvious by taking $u=v=\alpha=\beta=0$, we obtain $\mathrm{X}(0, \bullet) \leq 0$ and as $\mathrm{X}(0, \bullet) \geq 0$ proposition 5 , we get $\mathrm{X}(0, \bullet)=0$.

Let $u, v \in I$ and $\alpha, \beta \geq 0$ and with $0<\gamma=\alpha+\beta \leq 1$. Put $a=\frac{\alpha}{\gamma}$ and $b=\frac{\beta}{\gamma}$. Then $a+b=\frac{\alpha}{\gamma}+\frac{\beta}{\gamma}$ and so.

$$
\begin{aligned}
& X(\alpha u+\beta v, \bullet) \\
= & X(a \gamma u+b \gamma v) \leq a^{s} X(\gamma u, \bullet)+b^{s} X(\gamma v, \bullet) \\
= & a^{s} X(\gamma u+(1-\gamma) 0, \bullet)+b^{s} X(\gamma v+(1-\gamma) 0, \bullet) \\
\leq & a^{S} \gamma^{S} X(u, \bullet)+b^{S} \gamma^{S} X(v, \bullet)+(1-\gamma)^{S}\left(a^{S}+b^{S}\right) X(0, \bullet) \\
= & a^{S} \gamma^{S} X(u, \bullet)+b^{S} \gamma s X(, \bullet) \\
\leq & a^{S} X(u, \bullet)+b^{S} X(, \bullet)
\end{aligned}
$$

The following inequality is Hermite-Hadamard inequality for s-convex stochastic processes in the second sense.

Proposition 6. Let be a stochastic process $X: I \times \Omega \rightarrow \mathbb{R}$, it is integrate on at every point of the interval $(0,1) \times \Omega$. Then one has equality

$$
\int_{0}^{1} X(t u+(1-t) v, \bullet) d t=\int_{0}^{1} X((1-t) u+t v, \bullet) d t .(2.1)
$$

Proof. Let us denote $t^{*}=t u+(1-t) v, t \in[0,1]$. Then $d t^{*}=(u-v) d t$ and we have the change of variable

$$
\begin{aligned}
& \int_{0}^{1} X(t u+(1-t) v, \bullet) d t=\frac{1}{u-v} \int_{v}^{u} X\left(t^{*}, \bullet\right) d t^{*} \\
& =\frac{1}{v-u} \int_{u}^{v} X\left(t^{*}, \bullet\right) d t^{*} .
\end{aligned}
$$

Similarly, let us denote $k^{*}=((1-t) u+t v, \bullet), t \in[0,1]$. Then $d k^{*}=(u-v) d t$ and we have the change of variable

$$
\int_{0}^{1} X((1-t) u+t v, \bullet) d t=\frac{1}{v-u} \int_{u}^{v} X\left(k^{*}, \bullet\right) d k^{*}
$$

then equality is proved.

Theorem 6. If $X: I \times \Omega \rightarrow \mathbb{R}^{+}$stochastic process is $s$ convex in the second sense, $s \in(0,1)$ and $u, v \in I$, then one has the inequalities:

$$
\begin{aligned}
& 2^{s-1} X\left(\frac{u+v}{2}, \bullet\right) \leq \frac{1}{v-u} \int_{u}^{v} X(t, \bullet) d t \\
& \leq \frac{X(u, \bullet)+X(v, \bullet)}{s+1} .
\end{aligned}
$$

As $\mathrm{X}$ stochastic process is s-convex in the second sense, we have, for all $t \in[0,1]$

$$
X(\alpha u+(1-\alpha) v, \bullet) \leq \alpha^{s} X(u, \bullet)+(1-\alpha)^{s} X(v, \bullet)
$$

Integrating this inequality over $\alpha$ on $[0,1]$, we get

$$
\begin{aligned}
& \int_{0}^{1} X(\alpha u+(1-\alpha) v, \bullet) d \alpha \\
& \leq \int_{0}^{1} \alpha^{s} X(u, \bullet) d \alpha+\int_{0}^{1}(1-\alpha)^{s} X(v, \bullet) d \alpha \\
& =X(u, \bullet) \int_{0}^{1} \alpha^{s} d t+X(v, \bullet) \int_{0}^{1}(1-\alpha)^{s} d \alpha \\
& =\frac{X(u, \bullet)+X(v, \bullet)}{s+1}
\end{aligned}
$$


As the change of variable $t=\alpha u+(1-\alpha) v$ gives us that

$$
\int_{0}^{1} X(\alpha u+(1-\alpha) v, \bullet) d t=\frac{1}{v-u} \int_{u}^{v} X(t, \bullet) d t
$$

the second inequality in (2.2) is proved. To prove the first inequality in (2.2), we observe that for all $a, b \in I$ we have

$$
X\left(\frac{a+b}{2}, \bullet\right) \leq \frac{X(\alpha, \bullet)+X(b, \bullet)}{2^{s}}
$$

Now, let $a=t u+(1-t) v$ and $b=(1-t) u+t v$ with $t \in[0,1]$. Then we get by $(2.3)$ that:

$$
X\left(\frac{u+v}{2}, \bullet\right) \leq \frac{X(t u+(1-t) v, \bullet)+X((1-t) u+t v, \bullet)}{2^{s}}
$$

for all $t \in[0,1]$. Integrating this inequality on $[0,1]$ and take into accounting that the equality in Proposition 6, we deduce the first part of (2.2).

We are interested in pointing out some properties of this process as in the classical convex stochastic processes.

Theorem 7. Now, suppose that $X$ stochastic process is, $s \in(0,1]$ and consider the stochastic process $H(t, \bullet):[0,1] \times \Omega \rightarrow \mathbb{R}_{+}$

$$
H(\alpha, \bullet):=\frac{1}{v-u} \int_{u}^{v} X\left(\alpha t+(1-\alpha) \frac{u+v}{2}, \bullet\right) d t
$$

Let $X: I \times \Omega \rightarrow \mathbb{R}_{+}$stochastic process be a s-convex in the second sense on $I \times \Omega, s \in(0,1]$.

$\mathrm{i}$ : $\mathrm{H}$ is s-convex stochastic process in the secod sense. ii: We have the inequality:

$$
H(t, \bullet) \geq 2^{s-1} X\left(\frac{u+v}{2}, \bullet\right)
$$

iii: We have inequality:

$$
H(t, \bullet) \leq \min \left\{H_{1}(t), H_{2}(t)\right\}, t \in[0,1]
$$

where

$$
\begin{aligned}
H_{1}(t, \bullet)= & t^{s} \frac{1}{v-u} \int_{u}^{v} X(t, \bullet) d t+(1-t)^{s} X\left(\frac{u+v}{2}, \bullet\right) \\
& X\left(\alpha u+(1-\alpha) \frac{u+v}{2}, \bullet\right) \\
H_{2}(t, \bullet)= & \frac{+X\left(\alpha v+(1-\alpha) \frac{u+v}{2}, \bullet\right)}{s+1}
\end{aligned}
$$

And $t \in(0,1]$;

iv. If $\widehat{H}(t, \bullet):=\max \left\{H_{1}(t), H_{2}(t)\right\}, t \in[0,1]$, then

$$
\begin{aligned}
& \widehat{H}(t, \bullet) \leq t^{s} \frac{X(u, \bullet)+X(v, \bullet)}{s+1} \\
& +(1-t)^{s} \frac{2}{s+1} X\left(\frac{u+v}{2}, \bullet\right), t \in[0,1]
\end{aligned}
$$

Proof. i: Let $t_{1}, t_{2} \in[0,1]$ and $\alpha, \beta \geq 0$ with $\alpha+\beta=1$. We have succesily

$$
\begin{aligned}
& H(\alpha x+\beta y, \bullet) \\
& =\frac{1}{v-u} \int_{u}^{v} X\left((\alpha x+\beta y) t+[1-(\alpha x+\beta y)] \frac{u+v}{2}, \bullet\right) d t \\
& =\frac{1}{v-u} \int_{u}^{v} X\left(\begin{array}{l}
\alpha\left[x t+(1-x) \frac{u+v}{2}\right] \\
+\beta\left[y t+(1-y) \frac{u+v}{2}\right], \bullet
\end{array}\right) d t \\
& \leq \frac{1}{v-u} \int_{u}^{v} X\left(\begin{array}{l}
\alpha^{s} X\left[x t+(1-x) \frac{u+v}{2}\right] \\
+\beta^{s} X\left[y t+(1-y) \frac{u+v}{2}\right], \bullet
\end{array}\right) d t \\
& =\alpha^{s} H(x, \bullet)+\beta^{s} H(y, \bullet)
\end{aligned}
$$

which shows that $\mathrm{H}$ stochastic process is s-convex in the second sense.

ii: Suppose that $t \in(0,1]$. Then a simple change of variable $\theta=\alpha t+(1-\alpha) \frac{u+v}{2}$ gives us

$$
\begin{aligned}
& H(t, \bullet)=\frac{1}{\alpha(v-u)} \int_{\alpha u+(1-\alpha) \frac{u+v}{2}}^{\alpha v+(1-\alpha) \frac{u+v}{2}} X(\theta, \bullet) d \theta \\
& =\frac{1}{p-q} \int_{q}^{p} X(\theta, \bullet) d \theta
\end{aligned}
$$

where $p=\alpha v+(1-\alpha) \frac{u+v}{2}$ and $q=\alpha v+(1-\alpha) \frac{u+v}{2}$.

Applying the left hand side of Hermite-Hadamard inequality for second sense s-convex stochastic process, we get

$$
\frac{1}{p-q} \int_{q}^{p} X(\theta, \bullet) d \theta \geq 2^{s-1} X\left(\frac{p+q}{2}, \bullet\right)=2^{s-1} X\left(\frac{u+v}{2}, \bullet\right)
$$

Therefore inequality (2.4) is obtained.

iii: Applying the right hand side of Hermite-Hadamard inequality for second sense s-convex stochastic process, we also have

$$
\begin{aligned}
& \frac{1}{p-q} \int_{q}^{p} X(\theta, \bullet) d \theta \leq \frac{X(p, \bullet)+X(q, \bullet)}{s+1} \\
& =\frac{X\left(\alpha u+(1-\alpha) \frac{u+v}{2}, \bullet\right)+X\left(\alpha v+(1-\alpha) \frac{u+v}{2}, \bullet\right)}{s+1} \\
& =H_{2}(t, \bullet)
\end{aligned}
$$

for all $t \in[0,1]$.

Note that if $\mathrm{t}=0$, then Proof.

$$
X\left(\frac{u+v}{2}, \bullet\right)=H(0, \bullet) \leq H_{2}(0, \bullet)=\frac{2}{s+1} X\left(\frac{u+v}{2}, \bullet\right)
$$

is true as it is equivalent with

$$
(s-1) X\left(\frac{u+v}{2}, \bullet\right) \leq 0
$$

and we know that for $s \in(0,1], X\left(\frac{u+v}{2}, \bullet\right) \geq 0$. 
On the other hand, it is obvious that

$$
X\left(\alpha t+(1-\alpha) \frac{u+v}{2}, \bullet\right) \leq \alpha^{s} X(t, \bullet)+(1-\alpha)^{s} X\left(\frac{u+v}{2}, \bullet\right)
$$

for all $t \in[0,1]$ and $t \in I$.

Integrating this inequality on $[\mathrm{a}, \mathrm{b}]$ we get (2.5). iv: We have

$$
\begin{gathered}
\alpha^{s} X(u, \bullet)+(1-\alpha)^{s} X\left(\frac{u+v}{2}, \bullet\right) \\
H_{2}(t, \bullet) \leq \frac{+\alpha^{s} X(v, \bullet)+(1-\alpha)^{s} X\left(\frac{u+v}{2}, \bullet\right)}{s+1} \\
=\alpha^{s} \frac{X(u, \bullet)+X(v, \bullet)}{s+1}+(1-\alpha)^{s} \frac{2}{s+1} X\left(\frac{u+v}{2}, \bullet\right)
\end{gathered}
$$

for all $t \in[0,1]$.

On the other hand, we know that

$$
\frac{1}{v-u} \int_{u}^{v} X(t, \bullet) d t \leq \frac{X(u, \bullet)+X(v, \bullet)}{s+1}
$$

and

$$
(1-\alpha)^{s} X\left(\frac{u+v}{2}, \bullet\right) \leq(1-\alpha)^{s} \frac{2}{s+!} X\left(\frac{u+v}{2}, \bullet\right), t \in[0,1] .
$$

which gives us that

$$
H_{1}(t, \bullet) \leq \alpha^{s} \frac{X(u, \bullet)+X(v, \bullet)}{s+1}+(1-\alpha)^{s} \frac{2}{s+1} X\left(\frac{u+v}{2}, \bullet\right)
$$

and the theorem is proved.

Now, suppose that $\mathrm{X}$ stochastic process is $\mathrm{s}$ - convex in the second sense and integrable on $[u, v] \times \Omega$.

Consider the stochastic process

$F(\alpha, \bullet):=\frac{1}{(v-u)^{2}} \int_{u}^{v} \int_{u}^{v} X(\alpha s+(1-\alpha) t, \bullet) d s d t, \alpha \in[0,1]$.

The following theorem contains the main properties of this stochastic process.

Theorem 8. Let $X: I \times \Omega \rightarrow \mathbb{R}_{+}$s-convex in the second sense stochastic process be integrable on $I \times \Omega, s \in(0,1], u, v \in I$ with $u<v$.

i.

$$
F\left(x+\frac{1}{2}, \bullet\right)=F\left(\frac{1}{2}-x, \bullet\right)
$$

for all $s \in\left[0, \frac{1}{2}\right]$ and

$$
F(\alpha, \bullet)=F(1-\alpha, \bullet)
$$

for all $\alpha \in[0,1]$;

ii: $\mathrm{F}$ is s-convex stochastic process in the secod sense on $[0,1] \times \Omega$;

iii: We have the inequality:

$$
\begin{aligned}
& 2^{1-s} F(\alpha, \bullet) \geq F\left(\frac{1}{2}, \bullet\right) \\
& =\frac{1}{(v-u)^{2}} \int_{u}^{v} \int_{u}^{v} F\left(\frac{s+t}{2}, \bullet\right) d s d t, \alpha \in[0,1]
\end{aligned}
$$

iv: We have the inequality

$$
F(\alpha, \bullet) \geq 2^{s-1} H(\alpha, \bullet) \geq 4^{s-1} X\left(\frac{u+v}{2}, \bullet\right)
$$

for all $\alpha \in[0,1]$.

$\mathrm{v}$ : We have the inequality:

$$
F(\alpha, \bullet) \leq \min \left\{\begin{array}{l}
{\left[\alpha^{s}+(1-\alpha)^{s}\right] \frac{1}{v-u} \int_{u}^{v} X(s, \bullet) d s} \\
X(u, \bullet)+X(\alpha u+(1-\alpha) v, \bullet) \\
\frac{+X((1-\alpha) u+\alpha v, \bullet)+X(v, \bullet)}{(s+1)^{2}}
\end{array}\right\}
$$

for all $\alpha \in[0,1]$.

Proof. i. It is obvious owing to integrable properties. ii.

$$
\begin{aligned}
& F(\alpha x+\beta y, \bullet) \\
& =\frac{1}{(v-u)^{2}} \int_{u}^{v} \int_{u}^{v} X((\alpha x+\beta y) s+(1-(\alpha x+\beta y)) t, \bullet) d s d t \\
& =\frac{1}{(v-u)^{2}} \int_{u}^{v} \int_{u}^{v} X\left(\begin{array}{l}
\alpha(x s+(1-x) t, \bullet) \\
+\beta(y s+(1-y) t, \bullet)
\end{array}\right) d s d t \\
& \leq \frac{1}{(v-u)^{2}} \int_{u}^{v} \int_{u}^{v}\left[\begin{array}{l}
\alpha^{s} X(x s+(1-x) t, \bullet) \\
+\beta^{s} X(y s+(1-y) t, \bullet)
\end{array}\right] d s d t \\
& =\alpha^{s} F(x, \bullet)+\beta^{s} F(y, \bullet)
\end{aligned}
$$

iii. By the fact that $\mathrm{X}$ is s-convex stochastic process in the second sense on $I \times \Omega$. we have

$$
X\left(\frac{u+v}{2}, \bullet\right) \leq \frac{X(t u+(1-t) v, \bullet)+X((1-t) u+t v, \bullet)}{2^{s}}
$$

for all $t \in[0,1]$ and $u, v \in[a, \mathrm{~b}]$. Integrating this inequality on $[a, b]^{2}$ we have

$$
\begin{aligned}
& \int_{a}^{b} \int_{a}^{b} X\left(\frac{u+v}{2}, \bullet\right) d u d v \\
& \leq \frac{1}{2^{s}}\left[\begin{array}{l}
\int_{a}^{b} \int_{a}^{b} X(t u+(1-t) v, \bullet) d u d v+ \\
\int_{a}^{b} \int_{a}^{b} X((1-t) u+t v, \bullet) d u d v
\end{array}\right] .
\end{aligned}
$$

Since

$$
\begin{aligned}
& \int_{a}^{b} \int_{a}^{b} X(t u+(1-t) v, \bullet) d u d v= \\
& \int_{a}^{b} \int_{a}^{b} X((1-t) u+t v, \bullet) d u d v
\end{aligned}
$$

the (2.11) inequality and (2.12) equality gives us the desired result (2.8).

iv. First of all, let us observe that

$$
F(\alpha, \bullet):=\frac{1}{(v-u)} \int_{u}^{v}\left[\frac{1}{(v-u)} \int_{u}^{v} X(\alpha s+(1-\alpha) t, \bullet) d s\right] d t
$$

Now, for $\mathrm{y}$ fixed in $[u, v]$, we can consider the stochastic process $H_{t}:[0,1] \times \Omega \rightarrow \mathbb{R}$ given by 


$$
H_{t}(\alpha, \bullet):=\frac{1}{v-u} \int_{u}^{v} X(\alpha s+(1-\alpha) t, \bullet) d s
$$

As shown in the proof of Theorem 3, for $\alpha \in[0,1]$ we have the inequality

$$
H_{t}(\alpha, \bullet):=\frac{1}{p-q} \int_{q}^{p} X(\theta, \bullet) d \theta
$$

where $\quad p=\alpha v+(1-\alpha) t, q=\alpha u+(1-\alpha) s \quad$. Applying Hermite-Hadamard inequality for convex stochastic process in the second sense we get that

$$
\begin{aligned}
& \frac{1}{p-q} \int_{q}^{p} X(\theta, \bullet) d \theta \geq 2^{s-1} X\left(\frac{p+q}{2}, \bullet\right) \\
& =2^{s-1} X\left(\alpha \frac{u+v}{2}+(1-\alpha) t, \bullet\right)
\end{aligned}
$$

for all $\alpha \in(0,1)$ and $t \in[u, v]$. Integrating on $[u, v]$ over t, we easily deduce

$$
F(\alpha, \bullet) \geq 2^{s-1} H((1-\alpha), \bullet)
$$

for all $\alpha \in(0,1)$. As $F(\alpha, \bullet)=F(1-\alpha, \bullet)$ and by taking into inequality (2.4), the inequality (2.9) is proved for $\alpha \in(0,1)$. If $\mathrm{t}=0$ or $\mathrm{t}=1$, then this inequality also holds. We shall omit the details.

v. By the Definition of s-convex stochastic process in the second sense, we have

$$
X(\alpha u+(1-\alpha) v, \bullet) \leq \alpha^{s} X(u, \bullet)+(1-\alpha)^{s} X(v, \bullet)
$$

for all $u, v \in[a, b], \alpha \in[0,1]$. Integrating this inequality on $[u, v]^{2}$, we deduce the first part of the inequality (2.10).

Now, let us observe, by the second part of the HermiteHadamard inequality, that

$$
\begin{aligned}
& H_{t}(\alpha, \bullet):=\frac{1}{p-q} \int_{q}^{p} X(\theta, \bullet) d \theta \\
& \leq \frac{X(\alpha v+(1-\alpha) t, \bullet)+X(\alpha u+(1-\alpha) s, \bullet)}{s+1}
\end{aligned}
$$

where $p=\alpha v+(1-\alpha) t, q=\alpha u+(1-\alpha) s, \alpha \in[0,1]$.
Integrating this inequality on $[u ; v]$ over $\mathrm{t}$, we deduce

$$
F(\alpha, \bullet) \leq \frac{1}{s+1}\left[\begin{array}{l}
\frac{1}{v-u} \int_{u}^{v} X(\alpha v+(1-\alpha) t, \bullet) d t \\
+\frac{1}{v-u} \int_{u}^{v} X(\alpha u+(1-\alpha) s, \bullet) d s
\end{array}\right] .
$$

A simple calculation shows that

$$
\begin{aligned}
& \frac{1}{v-u} \int_{u}^{v} X(\alpha v+(1-\alpha) t, \bullet) d t \\
& =\frac{1}{r-1} \int_{l}^{r} X(\theta, \bullet) d \theta \leq \frac{X(r, \bullet)+X(l, \bullet)}{s+1} \\
& =\frac{X(u, \bullet)+X(\alpha v+(1-\alpha) u, \bullet)}{s+1}, \alpha \in(0,1),
\end{aligned}
$$

where $r=v, l=\alpha v+(1-\alpha) u, \alpha \in(0,1)$; and similarly,

$$
\begin{aligned}
& \frac{1}{v-u} \int_{u}^{v} X(\alpha u+(1-\alpha) t, \bullet) d t \\
& \leq \frac{X(u, \bullet)+X(\alpha u+(1-\alpha) v, \bullet)}{s+1}, \alpha \in(0,1),
\end{aligned}
$$

which gives, by addition, the second inequality in (2.10).

If $\mathrm{t}=0$ or $\mathrm{t}=1$, then this inequality also holds. We shall omit the details.

\section{References}

[1] K. Nikodem, on convex stochastic processes, Aequationes Mathematicae 20 (1980) 184-197.

[2] A. Skowronski, On some properties of J-convex stochastic processes, Aequationes Mathematicae 44 (1992) 249-258.

[3] A. Skowronski, On wright-convex stochastic processes, Annales Mathematicae Silesianne 9(1995) 29-32.

[4] D. Kotrys, Hermite-hadamart inequality for convex stochastic processes, Aequationes Mathematicae 83 (2012) 143-151.

[5] S.S. Dragomir and S. Fitzpatrick, The Hadamard's inequality for sconvex functions in the second sense, Demonstratio Math., 32 (4) (1999), 687-696.

[6] H. Hudzik and L. Maligranda, Some remarks on s-convex functions, Aequationes Mathematicae, 48 (1994), 100-111.

[7] S. Maden, M. Tomar and E. Set, s-convex stochastic processes in the first sense, Pure and Applied Mathematics Letters, in press.

[8] D. Kotrys, Hermite-hadamart inequality for convex stochastic processes, Aequationes Mathematicae 83 (2012) 143-151. 